\title{
DIETEXPERT - ANDROID APPLICATION FOR PERSONAL DIET CONSULTANT
}

\author{
Garvita Gehlot \\ Department of Computer Science and Engineering \\ Acropolis Institute of Technology and Research
}

\begin{abstract}
This application provides the user with a complex algorithm which can provide the user with a diet plan based on his/her characteristics like height, weight, BMI, gender etc. Everyone today dreams of healthy life cycle.

In Today's busy life healthy body is dream for everyone to have a proper balanced diet. $\mathrm{A}$ balanced diet is important because your organs and tissues need proper nutrition to work effectively. Without good nutrition, your body is more prone to disease, infection, fatigue, and poor performance. Children with a poor diet run the risk of growth and developmental problems and poor academic performance, and bad eating habits can persist for the rest of their lives. At the core of a balanced diet are foods that are low in unnecessary fats and sugars and high in vitamins, minerals, and other nutrients. The following food groups are essential parts of a balanced diet. Calories play a vital role in our growth and energy. A good diet can help you manipulate calorie intake based on your requirements. The proposed application will provide the user with a user-friendly User-Interface where they can create an account, manage their account and get the diet by the click of just one button. If the user is allergic to some kind of food, it also has the feature to contact an actual dietitian to consult. And there's also a page where the user can just read some interesting facts on health and human body. This application will save a lot of user's time by not actually visiting a dietitian and getting everything done on their phone.
\end{abstract}

\section{INTRODUCTION}

DietExpert is an android application is a provides a personalized diet to its users. It acts as a diet consultant similar to a real Dietitian. This system acts in a similar way as that of a dietitian. A person in order to know his/her diet plan needs to give some information to the dietitian such as its weight, height, gender etc. Similar way this system also provides the diet plan according to the information entered by the user. The system asks all data from the user and processes it to provide the diet plan to the user.

The project has a login page where the user is required to register his/her account and then they can use the app. Thus, the user does not need to visit any dietitian which also saves time and the user can get the required diet plan in just a click. The system will give more accurate results as it accepts the data entered by the user and processes it depending on some metrics already known to the application on the basis of which a diet plan is generated and ask the user if the user accepts the diet plan. If not accepted the system may also give and alternative diet plan. If a user wants to stay fit and eat healthy, he can surely follow the program provided to him. The Application also has a card for Health Facts on the home screen, which will provide all the general knowledge and some amazing facts on our human body and body parts. This Application can be a vital part of a user if he wishes to maintain his health and body perfectly and follow the diet plan \& the workout plan provided to the user.

\section{RELATED WORK}

In the Literature Survey part, we observed the main goal of our project which was to be made and started searching for published papers on it which will help us in building the application. We went across many IEEE \& Bayes Papers and found many papers which was some or the other way connected to our project based on health. We found many interesting papers as well as simple ones, we gathered the data from them. In the existing health care system, the primary requirement and disadvantage is physical presence of patient and doctor for every consultation.

In the existing diet consultant system, you have to hire a dietitian in order to get advice.

Also, there is a high chance of misinterpretation of data as well as occurrence of errors. Moreover, it is time consuming. With the increase in volume of patients in the health care institutes, traditional method of management has gone out of phase. As a result of this, an advanced Health Care Management System has been the demand of time. Some Systems were built directly for sole purpose of a single disease like Obesity, down syndrome etc. and some were general purpose applications, some projects website based and some were mobile application based. Our project was to be built on android so that people can get a good UI and also the app should be user-friendly. Some of the applications were paid-to-use and some were free, we wanted to build our project to be free to all. We started gathering information on the existing system and how it works and also a real dietitian works and calculates a diet based on a person's details like height, age, weight, gender etc. Even the internet helped us a lot for finding some basic formulas for calculating the diet and total calories. A person's diet totally depends upon what kind of activity he does in a day. If he/she has a hardworking job then they may lose more kcal as compared to other person who doesn't do much of hard work, so we have to calculate the Kcal based on the activity level of the person. The total calories to be consumed should be balanced proportion of macro nutrients like Proteins, Carbohydrates and Fats, with the ratio of $2: 2: 1$. In the existing health care system, the primary requirement and disadvantage is physical presence of 


\section{International Journal of Engineering Applied Sciences and Technology, 2021 \\ Vol. 5, Issue 12, ISSN No. 2455-2143, Pages 202-205 \\ Published Online April 2021 in IJEAST (http://www.ijeast.com)}

patient and doctor for every consultation. Also, there is a high chance of misinterpretation of data as 4 well as occurrence of errors. Moreover, it is cumbersome and time consuming. With the increase in volume of patients in the health care institutes, traditional method of management has gone out of phase. As a result of this, an advanced Health Care Management System has been the demand of time.

\section{DESIGN \& IMPLEMENTATION METHODS}

\subsection{Design:}

In this, we design the overview and implementation of the project was discussed. The modules discussed to be implemented are listed with some details. User Dashboard

- Profile

- Photo

- Goals

- Step Count

In the Design Process, we first designed the flow of events in which the application would work, which can be seen in the flowchart below In the Design Process, we first designed the flow of events in which the application would work, which can be seen in the flowchart below. We have to design a user registration system to be able to gain user information to calculate their details, we designed the registration and login system using Google's Firebase - A cloud-based system to store our data and authentication. It has a very easy to use interface and the Doc helps in implementing it. In the Registration process the app will take the details of users like age, height, weight, gender etc. and store it in the firebase database. There will be a dashboard card, where after the user logs in can view his details and also edit/ update the data. The Diet Plan card will give the user his data based on the details provided by the user while registering. All the calculations to get results was through research \& getting the right formulas. The Workout card was designed with the recycler view of the android's design system, it provides a list of items, we can change or update any items in the recycler view.

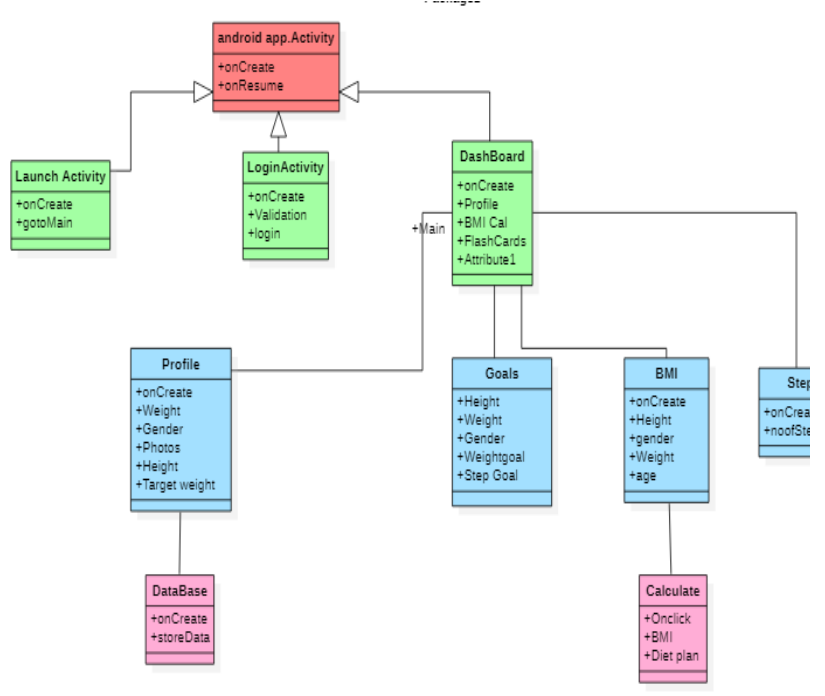

Fig1.Class Diagram of Application

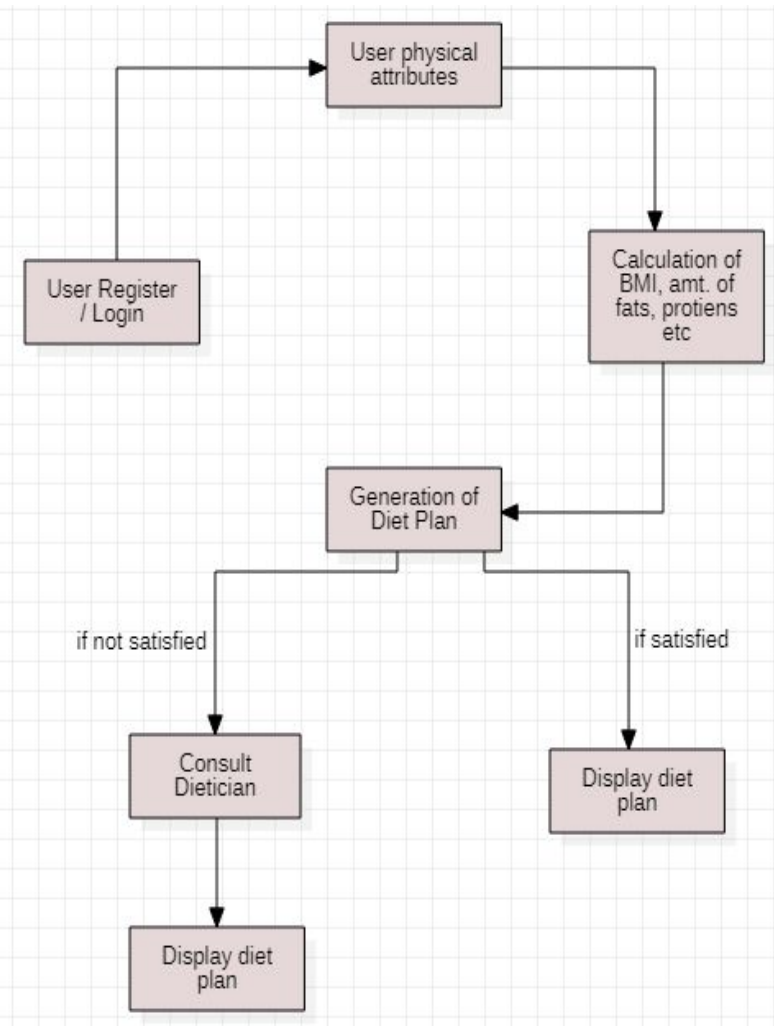

Fig2. Flow Diagram of application

\subsection{Implementation:}

Implementation is done with the help of the reference papers used and the internet, the application is built using Google's very own Android Studio. The Android Studio Docs helped us to implement certain design features to out app and also add API's for the firebase

database \& authentication. A few tutorials helped us to create a stable application that can work properly. The Formulas used for calculating the BMI (Body mass Index) and to calculate the total calories to intake was found on internet on some research.

\section{Formula for BMI:}

BMI = Weight / Height 2 Where, Weight is in Kilograms, Height is in Meters. Formula for calculating Maintenance Calories: Calories $=($ Weight $* 22) *$ Activity Multiplier Where, Weight is in Kilograms, Activity Multiplies refers to the amount of activity done in a day; it ranges from 1.2 for moderate work to 2.0 for extreme work done. Calculating Diet \& Providing Diet: The Maintenance calories of the users have to be calculated on the basis of his height, weight, age etc. The Maintenance Calories are calculated with the help of the formula given above, once the calories are calculated then the system decides if the user is in a under-weight, healthy or overweight category based on his BMI. The User is then suggested which type of diet program he / she should start, the user is still given the option to opt for the diet category like, Gain weight, Maintain Weight or Lose Weight. Based on 
Published Online April 2021 in IJEAST (http://www.ijeast.com)

the category selected the diet is provided by the application to the user. The Diet is based on the charts of diets calculated \& created by expert Dietitians and Nutritionists all over the globe. The User still has to option to consult a physical dietitian if he / she has some issues with the diet provided or has some food allergies or irritations, for example some people are Lactose Intolerant, so the dietitian will suggest some replacement for the lactose-based products in the diet.

\section{Application UI:}

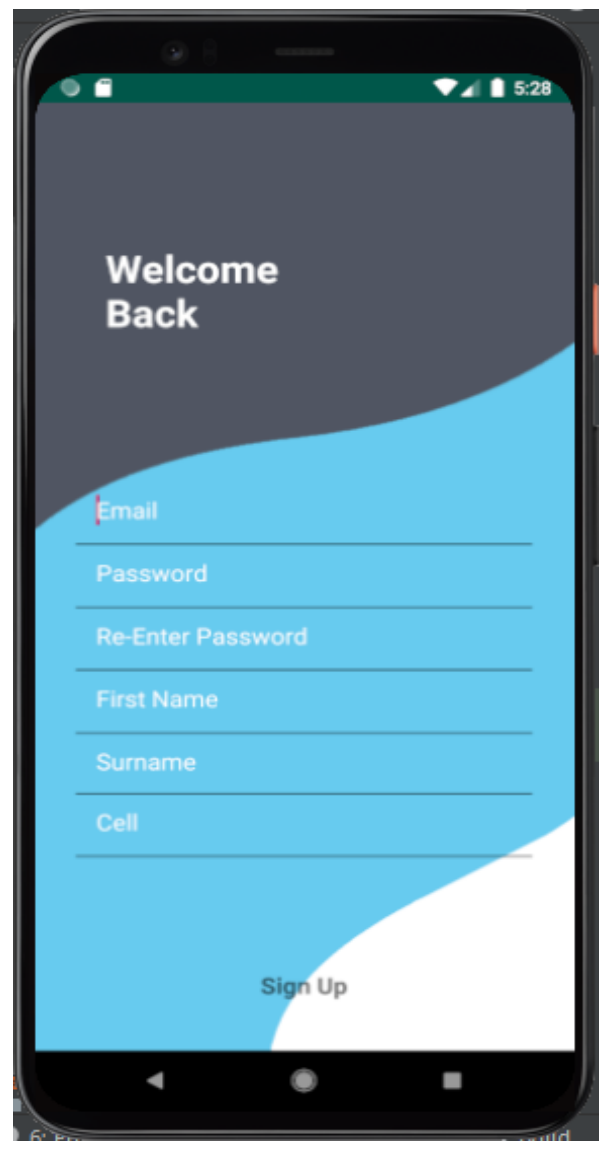

Fig3. Registration Screenshot

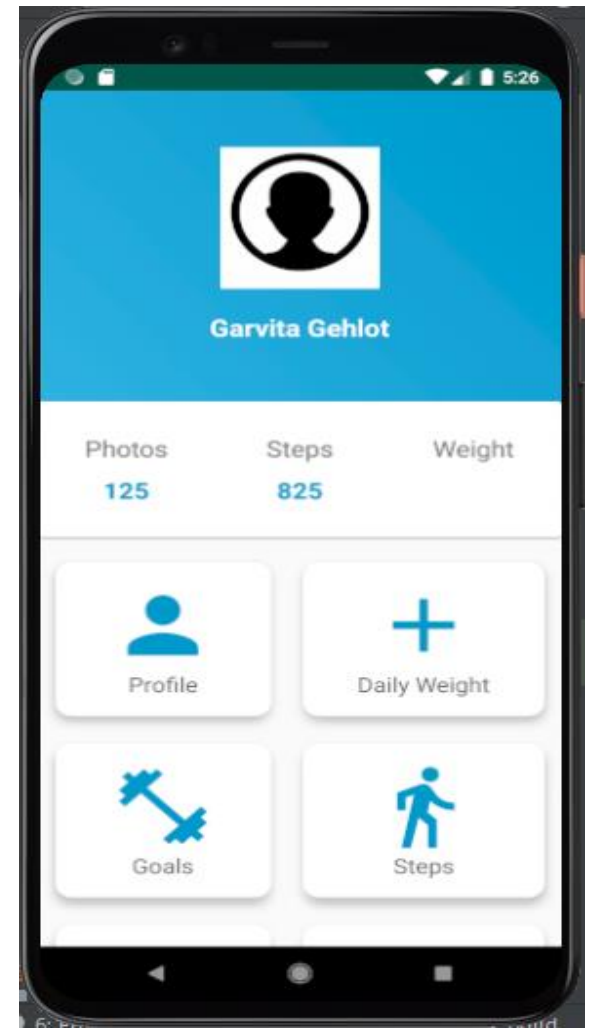

Fig4. Dashboard Screenshot

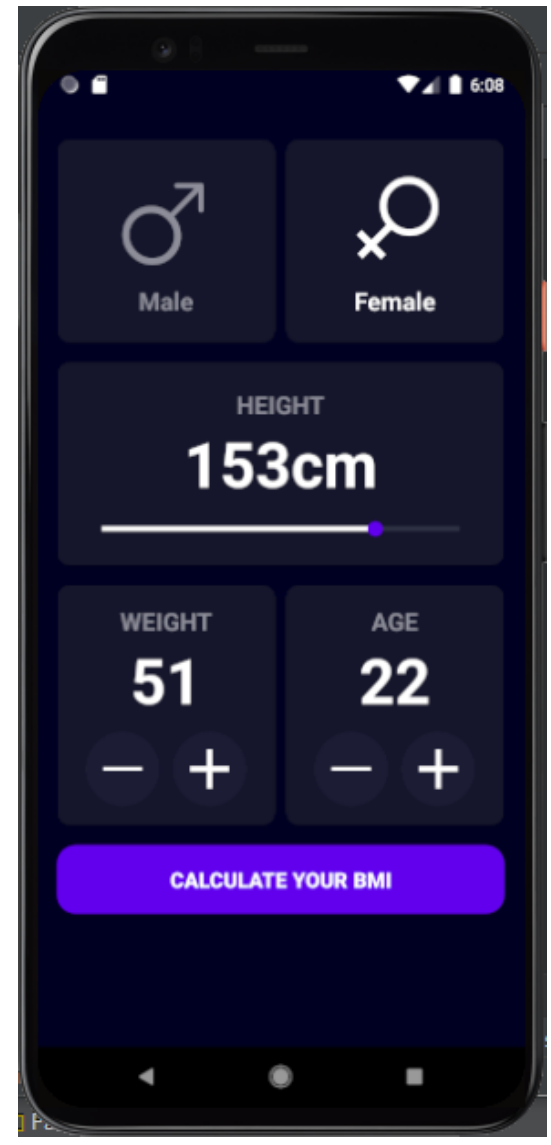

Fig5. BMI Calculator 


\section{International Journal of Engineering Applied Sciences and Technology, 2021 \\ Vol. 5, Issue 12, ISSN No. 2455-2143, Pages 202-205 \\ Published Online April 2021 in IJEAST (http://www.ijeast.com)}

\section{CONCLUSION}

With the proposed application on mobile phones, we will be able to bring the Dietitian App on the palm of every individual. The application can be deployed on the cloud by integrating different servers through the cloud in its future iterations. With respect to the feedback of the App users' further improvements can be incorporated within the system to make it more users friendly. In our approach for implementing this project is we have implemented a virtual dietitian using android. Our system comprises of main components such as of a user login and an admin login. The software system allows the user to create their profiles and upload all their details and their BMI is calculated by the system. The admin can check each user details and can remove faulty accounts. People who are in need of a serious health care but are busy with their schedules, can start using our application and start following the diet and workout programs. With the help of this application, user doesn't have to go to an actual dietitian he can have a dietitian in the palm of his hands.

\section{FUTURE SCOPE}

- This application can be further improved by feedback suggestions from the users.

- This application can be improved with the help of an expert nutritionist who can help us creating different types of programs for different classification of users.

- The project is easily extensible and can be improved by further incremental releases of the same.

- We plan to focus on improving the overall performance of the system. Also, interaction between guider and dietitian through video calling and secure prescription will be focused upon.

- Some more ways to achieve dietitian will be focused.

\section{REFERENCES}

\section{Published Survey Papers:}

[1]Prof. DV Chandran, Sayali Adarkar, Apoorva Joshi, Preeti Kajbajd," "Digital Medicine: An android based application for health care system", IRJET, Volume-4, 04Apr-2017.

[2]Hilde A.E Geraedts, Wiebren Zijlstra, Wei Zhang, Sophie L.W. Spoorenberg, "Home Based Exercise Program Driven By Tablet Application \& Mobility Monitoring, Public Health Research", Volume 14-E12, Feb-2017.

[3]Rodrigo Zenun Franco, Julie Anne Lovegrove, Rosalind Fallaize, Foustina Hwang, Popular Nutrition-Related Mobile Apps: A Feature Assessment, JMIR MHEALTH \& UHEALTH, Volume-4 Issue-3, Aug-2016.

[4] Oxford handbook of nutrition and dietetics edited by Joan Webster-Gandy, Angela Madden and Michelle Holdsworth
[5] Hong, S. M. and Kim, G. (2005). 'Web Expert System for Nutrition Counseling and Menu Management,' J Community Nutrition, $72,107-113$.

[6] Horn, W., C. Popow, S. Miksch, and A. Seyfang. (2002), 'Benefits of a Knowledge-based System for Parenteral Nutrition Support: a Report after 5 Years of Routin Daily Use,' Proceedings of the 15th European Conference on Artificial Intelligence (ECAI 2002), van Harmelen, F. (Ed.), 613-617.

[7] Khan,A. and Hoffmann,A.(2003). 'Building a case-based diet recommendation system without a knowledge engineer,' Artificial Intelligence in Medicine, 27, 155-179.

[8] Chen, Y. , Hsu, C. Y. , Liu, . L and Yang, S. (2012), 'Constructing a nutrition diagnosis expert system,' Expert System with Application, 39(2), 2132-2156

[9] Becerra-Fernandez, I., Gonzalez, A., \& Sabherwal, R. (2004). Knowledge management: Challenges, solutions and technologies. New Jersey: Pearson Education Inc.

[10]Balintfy JL. Menu planning by computer. Communications of the ACM. 1964;7(4):255-259. doi: 10.1145/364005.364087.

[11] Heart, Lung, and Blood Institute; National Institutes of Health, authors. Interactive menu planner.

[12] Eckstein E F. Menu planning by computer: the random approach. J Am Diet Assoc. 1967 Dec;51(6):529-33.

[13] Marling CR, Petot GJ, Sterling L. Integrating case-based and rule-based reasoning to meet multiple design constraints. Computational Intelligence. 1999;5(3):308-332. doi: 10.1111/0824-7935.00095.

[14] Petot G J, Marling C, Sterling L. An artificial intelligence system for computer-assisted menu planning. $J$ Am Diet Assoc. 1998 Sep;98(9):1009-14. doi: $10.1016 / \mathrm{S} 0002-82$ 